\title{
On Spatial Diversity for LoRaWAN: Experimental Evaluation of Performance of a Dual-Gateway Network With and Without Downlink
}

\author{
Konstantin Mikhaylov*† and Ari Pouttu* \\ ${ }^{*}$ Centre for Wireless Communications, University of Oulu, Oulu, Finland \\ ${ }^{\dagger}$ Faculty of Electrical Engineering and Communication, Brno University of Technology, Brno, Czech \\ konstantin.mikhaylov@oulu.fi
}

\begin{abstract}
The Low Power Wide Area Networks (LPWANs) is today seen as one of the key connectivity enablers for the massive Internet-of-Things (IoT) applications. In this paper, we experimentally study the performance of a LoRaWAN network composed of multiple end devices and two independentlyoperating gateways $(\mathrm{GW})$ for the case of uplink-only and uplinkdownlink traffic. Our results demonstrate that even though the spatial diversity due to the use of multiple GWs improves the performance of the network for the packet error rate, it still does not guarantee low packet loss under heavy uplink network traffic. Nonetheless, when the downlink traffic is present, a second GW operating in the uplink-only mode can dramatically increase the uplink packets delivery rate in the network. Therefore, we consider that spatial decoupling of receiver and transmitters, or addition of uplink-only GWs may become an efficient way to improve the performance of LoRaWAN networks with downlink traffic. Even though the limited laboratory environment might have affected the results of our measurements, we expect that many of the observed trends will also stand for the real-life LoRaWAN deployments. For this reason, the presented results might be interesting both for analysts and practitioners working in the field.
\end{abstract}

Keywords-LoRaWAN, Low Power Wide Area Network, LPWAN, gateway, uplink, downlink, performance, experiment.

\section{INTRODUCTION}

Today the establishment of the Internet-of-Things (IoT) is actively going on. Millions of new devices are being deployed every year to enable novel applications and exciting use cases. For this reason, today, the attention of both Industry and Academy is focused on the technologies underlying the IoT. Among these are the various wireless communication solutions, which are often seen as the key connectivity enablers for IoT[1] devices. Even though the commercial ultra-reliable Machine-Type Communication (uMTC) technologies [2] are mostly still in development, the massive Machine-Type Communication (mMTC) technologies are in commercial rollout already today.

Of all the variety of mMTC technologies, the Low Power Wide Area Networks (LPWANs)[4] show today tremendous growth[5]. Depending on the radio bands of operation, the LPWAN technologies can be subdivided into the two major groups: the ones operating in the licensed bands and the ones utilizing license-free bands. The former are represented primarily by the technologies adopted by $3 \mathrm{GPP}$, such as NBIoT or LTE-M. Among the latter ones, the LoRaWAN and SIGFOX are the most well-known. Of these two technologies, the LoRaWAN solution is reported[6] to be adopted more widely due to its higher technical and business flexibility.

Over the past few years, the LoRaWAN technology in general, and its different aspects have been excessively studied. Specifically, the propagation and coverage for LoRa-

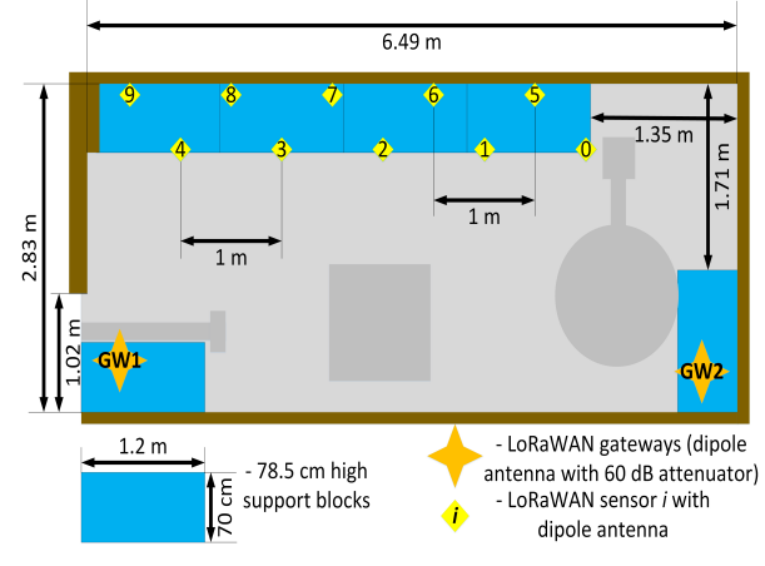

a. Experiment setup plan (the various laboratory equipment not used in the

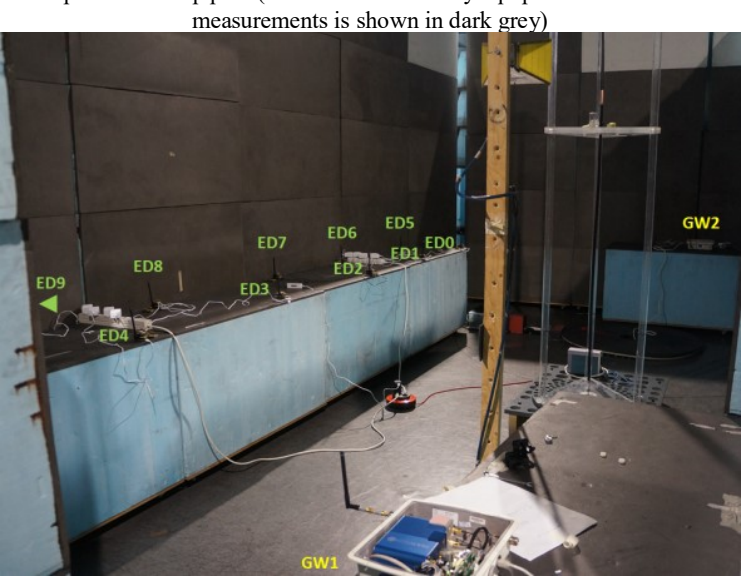

b. Illustration of the experiment environment and location of the individual EDs and GWs

Fig. 1. Experiment setup

modulated signals, representing the primary physical layer of LoRaWAN, have been investigated in [7][8]. The problem of interferences between the devices utilizing the same and different spreading factors has been analyzed in [9][10]. The restrictions imposed by the lack of resources on downlink communications [11] and even the possibility of interferences between uplink and downlink [12] have been demonstrated. Recently, the possibility of interferences between the adjacent frequency channels in LoRaWAN networks has also been shown [13].

In the current study, we extend the work started in [12]. In the previous paper, the extensive real-life experiments using commercial LoRaWAN equipment have been conducted to analyze the interferences between LoRa transmissions. One of the key findings of this study was the observation that the enablement of downlink transmission causes substantial degradation of the uplink data transfer performance in a 


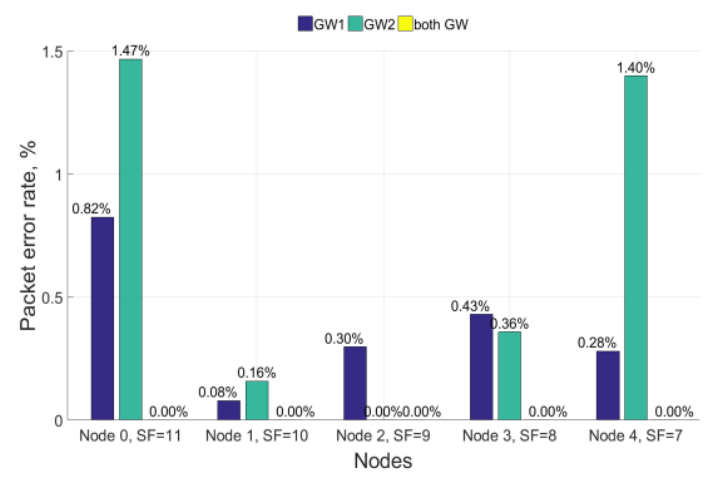

a. The PER of uplink transmissions for GW1, GW2 and the cumulative PER for the dual-GW network for five EDs configured to use unique secondary channels and SFs

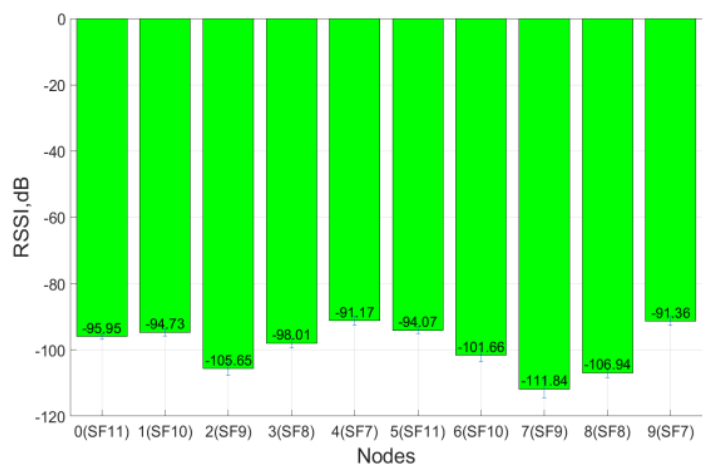

c. The RSSI of uplink transmissions by the EDs measured by GW1 (for the case of EDs using unique SF per pair and unique secondary channel per pair)



e. The PER of uplink transmissions for GW1, GW2 and the cumulative PER for the dual-GW network for ten EDs configured to use unique SF and unique secondary frequency channels per each two devices

Fig. 2. Selected experimental results for uplink-only transmissions

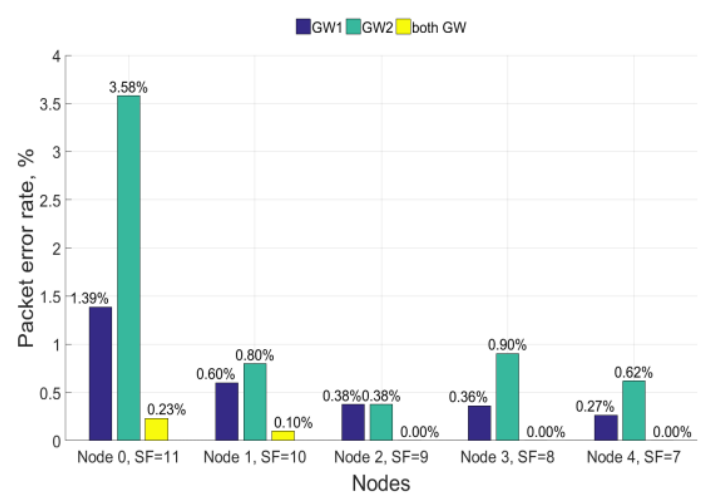

b. The PER of uplink transmissions for GW1, GW2 and the cumulative PER for the dual-GW network for five EDs configured to share secondary channels and use unique SFs



d. The RSSI of uplink transmissions by the EDs measured by GW2 (for the case of EDs using unique SF per pair and unique secondary channel per pair)

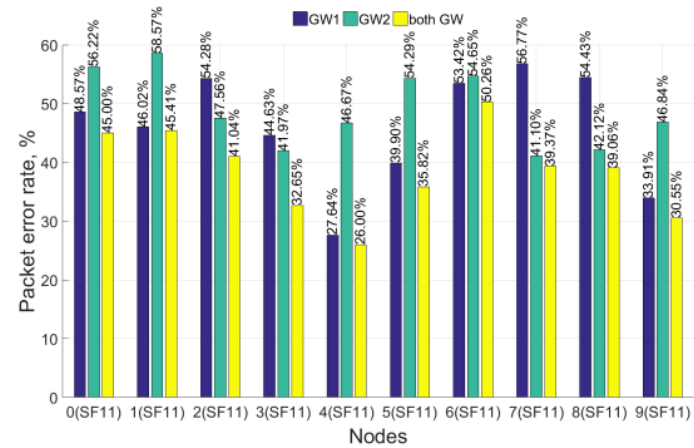

f. The PER of uplink transmissions for GW1, GW2 and the cumulative PER for the dual-GW network for ten EDs configured to use SF11 and share frequency channels single-gateway LoRaWAN network. As one of the means to address this challenge we have proposed to introduce another gateway $(\mathrm{GW})$ operating exclusively in receive, thus benefitting from spatial diversity. In the current paper, we validate this approach. Also, we experimentally check, using the state-of-the-art commercial equipment, how much does the spatial diversity improve the performance of a real-life LoRaWAN network both in uplink-only and in uplinkdownlink case. These results represent the major contribution of the current paper.

Note, that the experimental setup of our study is unique in a typical LoRaWAN, all GWs are connected to a single network server (NS), which typically deletes all packet duplicates received through different GWs. For our experiment, we have deployed two NS - one for each GW - which enables us to analyze how many (and which ones) each GW has received packets. Further, we analyze these data and estimate how many packets could have been received by a dual-GW network.

\section{EXPERIMENT SETUP}

The experimental setup and the operation of the devices for this study were similar to that in our earlier work [12]. Therefore, for the sake of brevity, in what follows, we will recap the most important aspects, while the other relevant details can be found from [12].

All the measurements were conducted in an anechoic chamber. During the tests, we have used five to ten end devices (EDs) constructed with CWC modular IoT hardware platform [14] equipped with commercial RN2483 LoRaWAN 




a. The PER of uplink transmissions for GW1 under downlink transmission through GW1 for five EDs using unique SFs and sharing the secondary frequency channels



c. The PER of uplink transmissions for GW1, GW2 and the cumulative PER under downlink transmission through GW1 for ten EDs using unique SFs and frequency channels per device pair

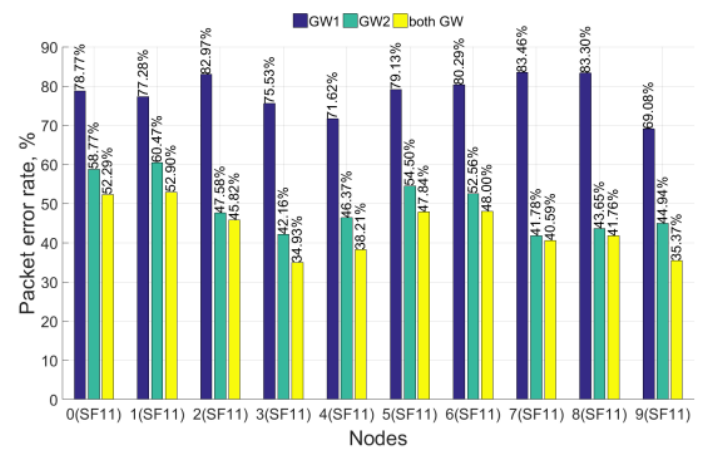

e. The PER of uplink transmissions for GW1, GW2 and the cumulative PER under downlink transmission through GW1 for ten EDs using SF11 and sharing the secondary frequency channels

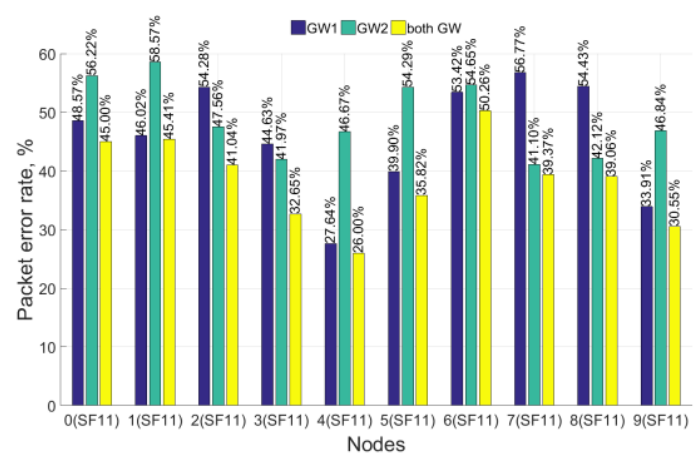

g. The PER of uplink transmissions for GW1, GW2 and the cumulative PER for uplink-only transmission for ten EDs using SF 11 and sharing the secondary frequency channels

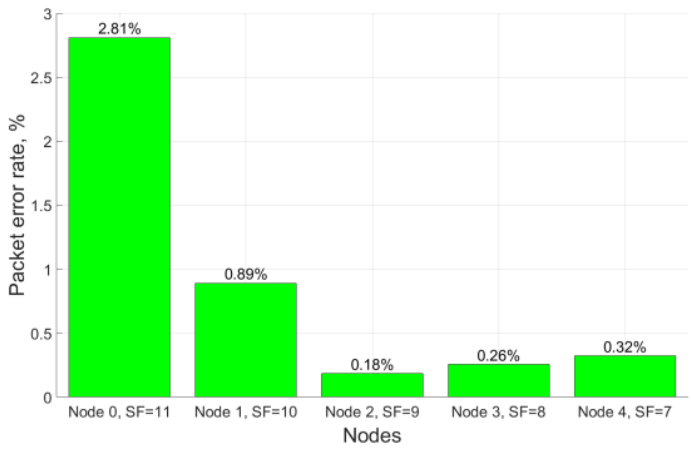

b. The PER of uplink transmissions for GW2 under downlink transmission through GW1 for five EDs using unique SFs and sharing the secondary frequency channels

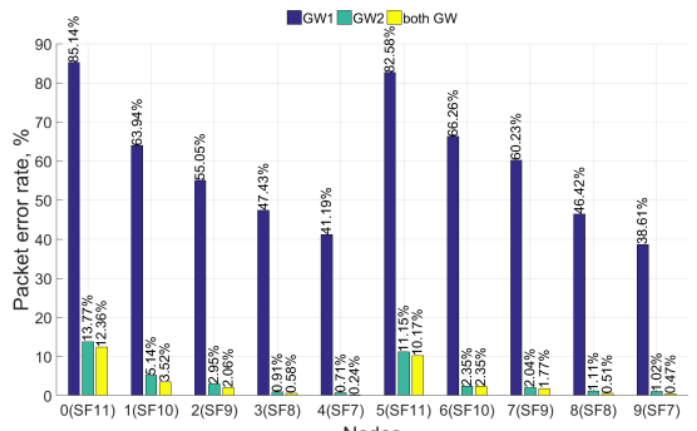

d. The PER of uplink transmissions for GW1, GW2 and the cumulative PER under downlink transmission through GW1 for ten EDs using unique SFs and sharing the secondary frequency channels



f. The PER of uplink transmissions for GW1, GW2 and the cumulative PER under downlink transmission through GW1 for ten EDs using SF7 and sharing the secondary frequency channels

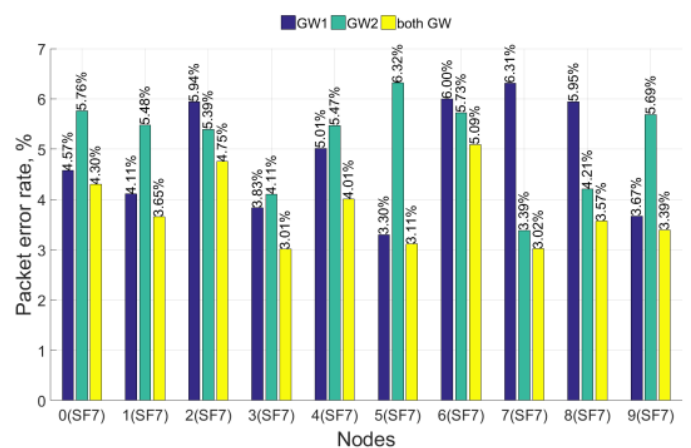

h. The PER of uplink transmissions for GW1, GW2 and the cumulative PER for uplink-only transmission for ten EDs using SF7 and sharing the secondary frequency channels

Fig. 3. Selected experiment results for uplink-downlink scenario 
transceiver modules from Microchip. As the GWs, we have used commercial MTCDT- LEU1 devices from MultiTech. The mutual location of the devices is depicted in Fig. 1. The principal difference of the current setup to the one in [12] is the addition of the second LoRaWAN GW - GW2.

The EDs were configured as class A LoRaWAN devices. The EDs were configured to send the 36-byte packets with a random uniformly distributed delay of 0.5 to 1.5 seconds between the packets. The EDs used three obligatory (i.e., 868.1, 868.3 and 868.5 MHz) EU LoRaWAN ("primary") channels and up to five additional ("secondary") channels in 869-870 MHz band. The duty cycle restriction for secondary channels was disabled. The transmit power for EDs was set to $14 \mathrm{dBm}$, and no adaptive data rate was enabled. The spreading factors for each ED were configured separately and changed from one experiment to another.

The GWs were configured to listen to all the channels used by the EDs. The NS was located right on the GW, and the special program for logging all the received packets, and the relevant data (e.g., the radio signal strength indicator (RSSI) or signal-noise ratio (SNR) estimate) was run on top of the NS. Additionally, a special application was developed and run on top of the NS of GW1 to generate a downlink packet in response to each uplink packet received.

Both EDs and GWs were equipped with half-wave dipole antennas. Besides, $60 \mathrm{~dB}$ attenuators were mounted between the antenna and the antenna port on the GWs to make the signal level more realistic.

In total, 22 different experiments (refer to [12] for further details) were conducted, each lasting over 75 minutes and composing 5000 - 20000 radio packets transmissions. The collected data were statistically processed using the developed MATLAB scripts.

\section{EXPERIMENTAL RESULTS}

\section{A. Uplink-only case}

The selected results for the uplink-only case are presented in Fig. 2. Subfigures a) and b) illustrate the packet error rate (PER) for the case of five LoRaWAN EDs with unique SFs operating in unique and shared secondary frequency channels, respectively. Subfigures c) and d) illustrate the radio signal strength (i.e., the RSSI) measured by each of the two GWs. Subfigures e) and f) reveal the PER for ten EDs configured to use unique channels and SFs per device pair, and using the same spreading factor (SF) and sharing the frequency channels, respectively.

From subfigures c) and d) one can see that the variation of the RSSI for the signals from different EDs for GW1 exceeded $20 \mathrm{~dB}$, while for GW2 it was below $5 \mathrm{~dB}$. This result is rather interesting, given that both of the GWs always had the line-ofsight towards the nodes in the first row, and often had line-ofsight towards second-row EDs. Nonetheless, the signal level of -90 to $-110 \mathrm{dBm}$ is well above the sensitivity limits of the SX1301 LoRa multi-channel baseband chip [15], which is installed (along with SX1255/SX1257) in MTAC-LoRa [16] card inside the Conduit GW used. Also, comparing the measured signal levels with the analytical signal-interference thresholds from the state-of-the-art literature (i.e., Table I in [10]) we can expect no negative effect of inter-SF interferences at GW2, and potential interference between from SF7 and SF10 to SF9 for GW1 for 10-node case only.
Nonetheless, the experimental results depicted in subfigures a) and b) show that even though all EDs are configured to use different SFs, several percents of packets still get lost. From subfigures a) and b) one can also see that the spatial diversity helps to improve the reliability of LoRaWAN data transfers from $94-98 \%$ for a single-GW setup to $99.7-100 \%$ for the double-GW network. Unfortunately, as this can be seen from subfigures e) and f), with the increase of the number of the nodes accessing the channel the PER substantially degrades, especially when all the nodes are configured to use the same SF. For these cases the availability of a second GW brings only a limited benefit, improving the PER by a few percents with respect to the PER of a GW in the best position for receiving the signals from this ED.

\section{B. Uplink and downlink case}

The selected results for the case when downlink transmission through GW1 was enabled are presented in Fig. 3. Subfigures a) and b) illustrate the PER for the case of five EDs operating with unique SFs and sharing the secondary channels for GW1 and GW2, respectively. Subfigures c) and d) depict the PER for individual GWs and the cumulative PER in the case of ten EDs using unique SF per device pair and operating either in unique secondary channels per pair of devices, or sharing all of them, respectively. Finally, subfigures e) and f) reveal the PER for individual GWs and the cumulative PER for ten EDs sharing the secondary frequency channels and operating with SF11 and SF7, respectively. Finally, for a reference subfigures g) and h) show the PER for the same configurations as e) and f), respectively, when no downlink traffic was present.

Comparing the PER for GW1 and GW2, one can see that the PER for GW2 is $40-90 \%$ lower than that for GW1. Comparing subfigures g) and e), or h) and f), one can see that the performance of GW2 is comparable to that for the case when no downlink traffic was present. Still, the PER of a double-GW system with one GW conveying the downlink is $2-20 \%$ beyond that of a dual-GW network with no downlink traffic present.

\section{DisCuSSION AND CONCLUSIONS}

To the best of our knowledge, the current paper is one of the first to provide some insights into the operation of a reallife multi-GW LoRaWAN network. Our results show that for the case when no downlink traffic was present, the addition of a second GW has substantially reduced the PER for a sparse network, and moderately improved the performance of a dense network with heavy traffic. Note that due to the very limited size of the anechoic chamber, we could not move the EDs far apart. This might have had affected the results of our experiments, e.g., by reducing the benefits of the spatial diversity of the GWs. Note also that the position of the GWs was not optimized in any way.

After enabling the downlink traffic for GW1, we have witnessed a dramatic increase in the number of uplink packets received by it. Still, the downlink of GW1 had almost no effect on the uplink performance of the second GW - GW2. Thus, this makes us conclude that the decoupling of an uplink receiver and a downlink transmitter, or, alternatively, the addition of an uplink-only GW might enable to improve the reliability and performance of a LoRaWAN network under the presence of downlink traffic. 
The possible reasons after such a strong effect of the downlink traffic on the performance of uplink reception for a GW are two-fold. First, if the GW's transceiver is half-duplex (i.e., does not support transmission and reception at the same time), this is easy to expect the receiver to get disconnected from the antenna by a switch, when the GW's transmitter is on, as this is discussed, e.g., in [17]. Obviously, this will prevent the GW from receiving anything in the uplink. Second, in the case, if the GW is full-duplex, the downlink transmission during RW1 might interfere with an uplink transmission in the same frequency channel (and, given the non-ideality of the frequency filters, may also affect the adjacent frequency channels - refer to, e.g., [13]). Unfortunately, due to the lack of technical details about the design of the commercial LoRaWAN GW used, we cannot define which of these two reasons has caused the observed behavior.

Nonetheless, the possibility of downlink transmissions affecting the uplink in a LoRaWAN needs to be carefully considered, studied further, and taken into account when modeling the LoRaWAN networks. Meanwhile, one of the possible ways to overcome this issue, as demonstrated in this paper, is the inclusion in the network of redundant GWs not conveying any downlink traffic. Such modification of the network topology, in our opinion, might be especially beneficial for the LPWANs deployed to serve the applications imposing quality-of-service requirements.

\section{ACKNOWLEDGMENT}

The measurements were undertaken in the context of the P2P-SMARTEST project, an Innovation Action funded by the H2020 Programme, contract number 646469. The analysis was done as a part of the LPWAN-evolution activity funded by the University of Oulu. This work is also supported by the Academy of Finland 6Genesis Flagship under Grant no. 318927. The work of K. Mikhaylov was also supported by the mobility project MeMoV, CZ.02.2.69/0.0/0.0/16_027/00083710, of Czech Ministry of Education, Youth and Sport, funded by European Social Fund and LPWAN-evolution activity funded by the University of Oulu.

\section{REFERENCES}

[1] Z. Qin, F. Y. Li, G. Y. Li, J. A. McCann, and Q. Ni, "Low-Power WideArea Networks for Sustainable IoT," IEEE Wireless Commun., vol. 26, no. 3, pp. 140-145, June 2019.

[2] M. Bennis, M. Debbah, and H. V. Poor, "Ultrareliable and LowLatency Wireless Communication: Tail, Risk, and Scale," Proc. IEEE, vol. 106 , no. 10 , pp. 1834-1853, Oct. 2018.
[3] M. R. Palattella et al., "Internet of Things in the 5G Era: Enablers, Architecture, and Business Models," IEEE J. Selected Areas Commun., vol. 34, no. 3, pp. 510-527, March 2016.

[4] U. Raza, P. Kulkarni, and M. Sooriyabandara, "Low Power Wide Area Networks: An Overview," IEEE Commun. Surv. \& Tut., vol. 19, no. 2, pp. 855-873, Secondquarter 2017.

[5] IoTNow, LPWAN: The fastest growing IoT communication technology, available: https:/www.iot-now.com/2018/10/29/89895lpwan-fastest-growing-iot-communication-technology/

[6] IHS Markit, Connectivity technologies, available: https://cdn.ihs.com/www/pdf/IHS\%20Markit\%20-

$\% 20$ Connectivity $\% 20$ Technology\%20Competition\%20Drivers\%20an d\%20Influencers.pdf

[7] J. Petäjäjärvi, M. Pettissalo, K. Mikhaylov, A. Roivainen, and T. Hänninen, "On the Coverage of LPWANs: Range Evaluation and Channel Attenuation Model for LoRa Technology," in Proc. Int. Conf. Intelligent Trasportation Syst. Telecommun., Dec. 2-4 2015, Copenhagen, Denmark, pp. 55-59.

[8] L. Vangelista, "Frequency Shift Chirp Modulation: The LoRa Modulation," IEEE Signal Proc. Let., vol. 24, no. 12, pp. 1818-1821, Dec. 2017.

[9] T. Elshabrawy and J. Robert, "Analysis of BER and Coverage Performance of LoRa Modulation under Same Spreading Factor Interference," in Proc. 29th IEEE Annu. Int. Symp. Personal, Indoor Mobile Radio Commun., 9-12 Sept. 2018, Bologna, Italy, pp. 1-6.

[10] D. Croce, M. Gucciardo, S. Mangione, G. Santaromita, and I. Tinnirello, "Impact of LoRa Imperfect Orthogonality: Analysis of Link-Level Performance," IEEE Commun. Let., vol. 22, no. 4, pp. 796799, April 2018.

[11] A. Pop, U. Raza, P. Kulkarni, and M. Sooriyabandara, "Does Bidirectional Traffic Do More Harm Than Good in LoRaWAN Based LPWA Networks?," in Proc. IEEE Global Commun. Conf., Singapore, Singapore, 4-8 Dec. 2017, pp. 1-6.

[12] K. Mikhaylov , J. Petäjäjärvi, and A. Pouttu, "Effect of Downlink Traffic on Performance of LoRaWAN LPWA Networks: Empirical Study," in Proc. 29th IEEE Annu. Int. Symp. Personal, Indoor Mobile Radio Commun., 9-12 Sept. 2018, Bologna, Italy, pp. 1-6.

[13] M. Chiani and A. Elzanaty, "On the LoRa Modulation for IoT: Waveform Properties and Spectral Analysis," IEEE Internet Things J., in press.

[14] K. Mikhaylov and J. Petäjäjärvi, "Design and Implementation of the Plug\&Play enabled Flexible Modular Wireless Sensor and Actuator Network Platform," Asian J. Control, vol. 19, no. 5, pp. 1-21, Sept. 2017.

[15] Semtech, SX1301, datasheet, available: https://www.semtech.com/uploads/documents/sx1301.pdf

[16] Multitech, LoRaWAN Troubleshooting guide, available http://www.multitech.net/developer/wpcontent/uploads/downloads/2018/03/LoRaWANTroubleShootingGuid e-030920181.pdf

[17] V. Di Vincenzo, M. Heusse, and B. Tourancheau, "Improving Downlink Scalability in LoRaWAN," in Proc. Int. Conf. Commun., Shaghai, China, 20-24 May 2019, pp. 1-6. 